
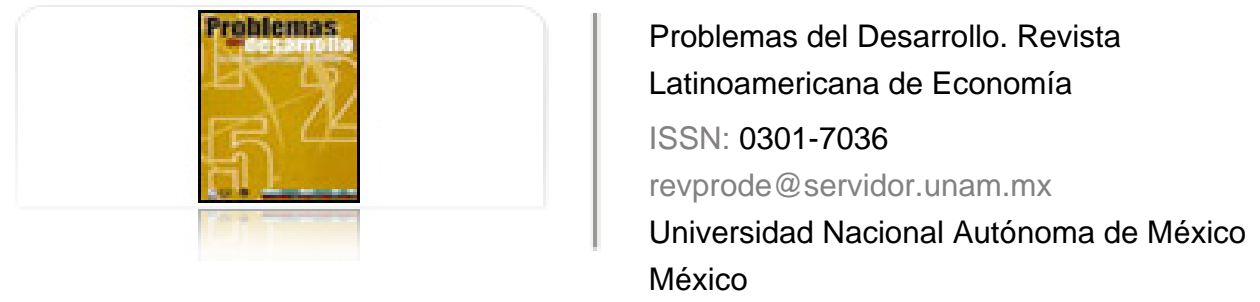

González García, Juan; Meza Lora, José Salvador Shenzhen, zona económica especial: bisagra de la apertura económica y el desarrollo regional chino Problemas del Desarrollo. Revista Latinoamericana de Economía, vol. 40, núm. 156, enero-marzo, 2009, pp. 101-124

Universidad Nacional Autónoma de México

Distrito Federal, México

Disponible en: http://www.redalyc.org/articulo.oa?id=11820096005

Cómo citar el artículo

- Número completo

- Más información del artículo

Página de la revista en redalyc.org

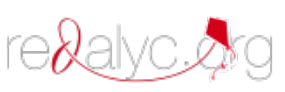

Sistema de Información Científica

Red de Revistas Científicas de América Latina, el Caribe, España y Portugal Proyecto académico sin fines de lucro, desarrollado bajo la iniciativa de acceso abierto 


\title{
SHENZHEN, ZONA ECONÓMICA ESPECIAL: BISAGRA DE LA APERTURA ECONÓMICA Y EL DESARROLLO REGIONAL CHINO
}

\author{
Juan González García* \\ José Salvador Meza Lora**
}

Fecha de recepción: 20 de octubre de 2008. Fecha de aceptación: 10 de febrero de 2009.

\section{Resumen}

En este trabajo se analiza el fenómeno reciente de las zonas económicas especiales (ZEE) en el mundo. Se presenta el caso de Shenzhen, ciudad de Guangdong, República Popular China (RPC), porque aquí se ha logrado el resultado más exitoso de la estrategia de reforma económica neoinstitucional y de apertura externa de ese país en las últimas tres décadas. Tal resultado indica la articulación de una estrategia gradual y de largo plazo, que le ha permitido a China una inserción internacional exitosa. En contraparte, se estudia el caso de México, nación que ha impulsado un tipo de reforma económica y apertura externa sin los resultados económicos y regionales esperados, por lo que se concluye sobre la necesidad de conocer el caso de dicha ZEE si es que se opta por una estrategia similar a la china.

Palabras clave: desarrollo económico, ZEE, apertura externa, reforma económica, Estado, mercado y desarrollo regional.

* Profesor-investigador de la Facultad de Economía de la Universidad de Colima y del Centro Universitario de Estudios e Investigaciones sobre la Cuenca del Pacífico. Correo electrónico: gogar@cgic.ucol.mx.

** Profesor de carrera nivel C de tiempo completo, Universidad Autónoma de Baja California, Unidad Tijuana. Correo electrónico: jsml@uabc.mx. 
Abstract

This study analyzes the recent phenomenon of special economic zones (SEZ) in the world. It presents the case of Shenzhen, a city in Guangdong, in the People's Republic of China (PRC), because a more successful result has been achieved there in the strategy of neo-institutional economic reform and the external opening of that country in the last three decades. This result indicates the articulation of a gradual and long-term strategy, which has enabled China to insert itself successfully into the international economy. In contrast, the case of Mexico is studied, a nation which has pursued a type of economic reform and external opening without the expected economic and regional results. We therefore conclude that it is necessary to understand the case of the so-called SEZ if a strategy similar to China's is to be embarked upon.

Key words: economic development, SEZ, external opening, economic reform, State, market and regional development.

\section{Résumé}

Dans ce travail, il est fait l'analyse du phénomène récent des zones économiques spéciales (ZEE) dans le monde. Il y est présenté le cas de Shenzhen, ville de Guangdong, République Populaire de Chine (RPC), parce qu'il s'y est obtenue la plus grande réussite de la stratégie de réforme économique néoinstitutionnelle et d'ouverture sur l'extérieur de ce pays, au cours des trois dernières décennies. Un tel résultat révèle l'articulation d'une stratégie graduelle à long terme qui a permis à la Chine de s'insérer dans l'économie mondiale avec succès. En contrepartie l'étude traite le cas du Mexique, nation qui a impulsé un type de réforme économique et d'ouverture extérieure sans atteindre les résultats économiques et régionaux escomptés, ce pourquoi la conclusion porte sur le besoin de connaître le cas de la ZEE mentionnée si on opte pour une stratégie similaire à celle de la Chine.

Mots clés: développement économique, ZEE, ouverture extérieure, réforme économique, État, marché et développement régional.

\section{Resumo}

Neste trabalho se analisa o fenômeno recente das zonas econômicas especiais (ZEE) no mundo. Apresenta-se o caso de Shenzhen, cidade de Guangdong, Republica Popular da China (RPC), porque aqui se alcançou o resultado mais exitoso da estratégia de reforma econômica neo-institucional e de abertura externa desse país nas três ultimas décadas. Esse resultado indica a articulação de uma estratégia gradual e de longo prazo que permite à China uma integração internacional exitosa. Em compensação se estuda o caso do México, nação que tem impulsado um tipo de reforma econômica e abertura externa sem os resultados econômicos e regionais esperados, pelo que inclui sobre a necessidade de conhecer o caso da ZEE se é o que se decide por uma estratégia semelhante a da China.

Palavras chave: desenvolvimento econômico, ZEE, abertura externa, reforma econômica, Estado, mercado e desenvolvimento regional.

\section{Desarrollo}




\section{Introducción}

crecimiento económico alcanzado por China durante los últimos 30 años
$(1978-2008)$ no tiene parangón en la historia económica mundial de los siglos
XIX y XX, debido a los logros económicos alcanzados: una tasa de crecimiento promedio anual cercano a $10 \%$ en su Producto Interno Bruto (PIB); el valor real de su PIB se ha incrementado en 49 veces desde 1978, con los 2.96 billones de dólares de 2008, y su comercio exterior ha crecido de manera extraordinaria con una tasa anual de $25 \%$ entre 2000 y 2008 (People Daily, 2008).

Su participación en el valor del comercio mundial de bienes se incrementó de $1.78 \%$ de 1978 a $6.3 \%$ en 2007 , ocupando también en este rubro el tercer lugar en el mundo; $17.5 \%$ del crecimiento del PIB mundial se debe a la dinámica económica de este país; aunque participa con sólo 5\% del total de las exportaciones manufactureras mundiales; contribuye con $29 \%$ en el crecimiento total de la producción manufacturera; en 2008 siguió siendo el país subdesarrollado que recibió mayor monto de inversión extranjera directa (IED), con alrededor de 71 mil millones de dólares, procedentes de 192 países con 53,000 empresas extranjeras establecidas en su territorio; según la revista Fortune en 2008; de las 500 empresas más importantes a escala internacional, 450 han invertido en el territorio chino y 30 de ellas han establecido ahí sus oficinas centrales (Xinhua, 2009).

Lo anterior ha despertado el interés de especialistas y no especialistas, quienes se preguntan acerca de las causas que han originado, por ejemplo, esa tasa de crecimiento económico y, en general, esos resultados económicos, que en lo social (disminución de la pobreza extrema en $80 \%$ de la población pobre de 1949) parecen indicar que China está inscribiéndose en los países con mayores posibilidades para alcanzar su desarrollo económico en este siglo XXI.

Obviamente no hay una respuesta única, pues sin duda existen muchas causas que han incidido para el logro de su crecimiento. De las diversas explicaciones, coincidimos con quienes señalan que dos han sido las razones principales que lo explican: la reforma económica y su inserción a la economía mundial (González, 2003:33; Yongtu Long, 2005:1; Cornejo, 2007; 192; Cornejo, 2008:10).

En efecto, entre 1949 y 1978 China fue una economía centralmente planificada con periodos combinados de crecimiento y años de decrecimiento económico, pero con indicadores bajos en el desarrollo económico y el bienestar social. Durante la revolución cultural (1966-1976, aunque oficialmente concluyó en 1969) el deterioro de la economía china tocó fondo, el crecimiento económico se torno negativo y los índices de pobreza se incrementaron de manera notable (González, 2002:35). Des- 
pués de la muerte de Mao Tse Dong, en 1976, y luego de un periodo de inestabilidad y cambio durante el gobierno de Hua Huofeng, se abandonó por completo el modelo económico soviético y se aplicó un nuevo modelo de desarrollo económico, orientado hacia el exterior, basado en la combinación mecanismo de mercado-Estado, donde el segundo ha mantenido una firme dirección e intervención en el sistema económico, fundamentado en un tipo de reforma económica neoinstitucional (REN) y la apertura externa (AE), combinada con estrategias de focalización del desarrollo regional desequilibrado, para propiciar la sinergia del modelo económico entre lo local y lo global (Zhou\& Chuah, 2002), en busca de propiciar que el desarrollo regional se dé de manera más equilibrada (Cornejo, 2007: 95).

Fue Deng Xiaoping quien lanzó esta propuesta de reforma y apertura a la economía mundial en 1978, pues estaba convencido de que "pobreza no es socialismo" y de que salir de ella debería ser la principal prioridad del país. Desde su perspectiva, cualquier medida que condujera a ese objetivo debería ser considerada correcta. Su filosofía: "la práctica como último criterio de verdad" y el eslogan "no importa el color del gato sino que cace ratones" son una síntesis del pragmatismo de su pensamiento. El eje fundamental de su REN era transformar la economía china de planificada a una socialista de mercado.

En el periodo 1949-1978 el Estado era el propietario de la tierra, de los medios de producción y del producto generado, pero a partir de 1979, con la REN y su aplicación, se introdujo el mecanismo de mercado en la economía de manera gradual y dio pie a la aparición de los agentes económicos privados. En la actualidad, el sector privado contribuye de manera importante en la economía china con una participación cercana a 70\% del PIB, esperándose que en 2010 este sector participe con tres cuartas partes de la producción en bienes y servicios de toda China (Long Yongtu, 2006:1).

La segunda razón que explica el crecimiento en China tiene que ver con su política de puertas abiertas (PA), que definió su manera particular de insertarse en la globalización económica. Para los dirigentes chinos de la gran reforma de 1978 era imposible construir una China moderna y una economía capaz de impulsar el desarrollo económico si continuaban con la autarquía. En razón de lo anterior se inició la aplicación de una política de PA con la finalidad de aprovechar los beneficios de la globalización. La aplicación acertada de políticas de integración a la economía mundial le permitiría ser receptora de capital, tecnología y recursos humanos calificados (Bhaskar Goswami, 2007).

Ambas estrategias, la reforma económica y la inserción a la globalización económica, desde el cambio de modelo de desarrollo económico de 1978, están inter- 
conectadas y no actúan de manera separada, es decir, forman parte de la estrategia integral china del modelo de desarrollo económico de orientación externa: sin la REN gradual, China jamás se hubiese abierto al exterior; sin la AE, también gradual, la REN no hubiera sido viable, y como consecuencia quizá el modelo económico chino sería un fracaso más, como muchos de los casos de países que pensaron que sólo cambiando su modelo de desarrollo económico era suficiente para buscar el desarro1lo. Por tanto, hablar de la política de puertas abiertas en China es hablar de la REN y particularmente del tipo de reforma, con el gradualismo como característica fundamental de la misma (Yongtu Long, 2005:2). Desde nuestra perspectiva de análisis, planteamos la hipótesis de que la REN de China y su política de puertas abiertas han sido determinantes para el éxito de su reinserción a la economía internacional con efectos regionales internos.

Por tanto, revisar la estrategia particular de China, en lo referente a su política de puertas abiertas y de AE gradual de su territorio a la economía internacional, puede ser aleccionadora para países en desarrollo que están impulsando o pretendan crear zonas económicas especiales, pensando en que con la sola creación de éstas lograrán generarse engranajes que arrastren a toda su economía para, mediante el desarrollo de sus regiones, contribuir al desarrollo económico nacional (Claus, 2000:5).

El artículo constará de cuatro partes. En la primera, describiremos de manera breve la evolución de las ZEE en el mundo, pues consideramos que son el punto de referencia básico para comprender cualquier estrategia, sobre todo de los países subdesarrollados para intentar insertarse exitosamente en la economía internacional; en la segunda analizaremos la estrategia global de inserción de la República Popular China (RPC) a la economía internacional de fines de la década de los setenta del siglo XX; en la tercera expondremos el caso de la ZEE de Shenzhen, que es el caso más exitoso de las ZEE de China en las dos décadas recientes, y en la última parte se revisará brevemente el caso de México, uno de los países pioneros en América Latina en impulsar la creación de zonas francas en su territorio, mismas que fueron canceladas en 1994 con la entrada en vigor del Tratado de Libre Comercio de América del Norte (TLCAN) con Estados Unidos y Canadá; y que también cambió su modelo de desarrollo económico en 1982, buscando entre otros objetivos una nueva forma de integración a la economía mundial. Al final del artículo se incluirá las conclusiones que reflejen lo expuesto y prospecten las implicaciones de este tipo de estrategia dual, de reinserción internacional con integración regional nacional. 


\section{Las ZEE en el mundo}

La creación de las ZEE en el mundo no es un dato reciente, su surgimiento obedece a la aplicación de una política industrial-comercial de un país, sea desarrollado o no, para impulsar a una determinada región o regiones, o a un determinado sector o ramas industriales económicas, o a la búsqueda de una política de inserción internacional. La clave es que dicha política esté contenida dentro de una estrategia global que articule el desarrollo regional nacional y local, con una estrategia congruente de inserción internacional en el corto, mediano y largo plazos (Park, 1997:15).

Al privilegiar la creación de estas ZEE, obviamente no se previó que éstas fueran a generar problemas sociales ni desequilibrios ambientales, como es una constante en la mayoría de ellas en la actualidad, sino que se ponderaron más sus efectos positivos y es parte de la preocupación de las autoridades nacionales, estatales y municipales de todos los países en los que existen dichas ZEE (Lumes, 2007:15).

Desde el punto de vista de la teoría del desarrollo económico (si se acepta el supuesto de que la teoría del desarrollo regional está anidada en ésta), sus fundamentos se encuentran en las formulaciones de Francois Perroux, Albert Hirsckman y Raúl Prebisch (González, 2007:24-33), quienes postulan que para lograr el desarrollo de un país se debe establecer y aplicar una estrategia de impulso a una determinada región económica, que permita articular a las demás regiones, estableciendo eslabonamientos industriales y comerciales, cadenas productivas positivas; generar y ampliar mercados internos y permitir el surgimiento de sectores y una economía de enclave para que los efectos positivos se propaguen al resto de las regiones, de tal manera que se consoliden capacidades competitivas dinámicas, mediante políticas de desarrollo regional que brinden facilidades fiscales, comerciales, industriales, demográficas y educativas.

Los antecedentes históricos de la creación de las primeras ZEE se encuentran en Europa Occidental de mediados del siglo XX (Hernández Pedraza et al.; 2005: 89). En efecto, la primera ZEE se estableció en Irlanda en 1959, en la ciudad de Shannon, con la finalidad de establecer un cluster industrial para que las corporaciones transnacionales tuvieran mayores facilidades de acceso a la importación de insumos industriales para la exportación de sus productos; en Asia, la primera ZEE se erigió en Taiwán en la ciudad de Kaoshiung a mediados de los sesenta (Ota, 2003:8) y al final de dicha década existían 10 ZEE, sobre todo en Asia y América Latina; para mediados de los años setenta existían casi 80 ZEE en 25 países y entre 1975 y 2005 — sobre todo

a partir de la caída del muro de Berlín en 1989 y el desmembramiento de la ex Unión Soviética en 1991- la cifra se elevó a aproximadamente 3,000 ZEE en 120 países, la 
mayoría de éstas en Asia (Hernández Pedraza, 2005:90). El gobierno de India aprobó en 2007 la creación 36 ZEE más, por lo que en dicho país suman 339, concentrando más de $10 \%$ de todas la ZEE del mundo, cuya cifra aproximada asciende a 3,036.

La característica de estas ZEE es que ofrecen incentivos a los inversionistas externos, expectativas de rendimientos económicos altos, cesión de derechos sobre terrenos y concesiones para explotar los recursos del país huésped, mercados de procesamiento de productos para reexportar, exenciones fiscales, condiciones infraestructurales, facilidades administrativas, mano de obra barata y calificada y expectativas de crecimiento económico para el desarrollo de los mercados internos. De ellas, se espera que generen efectos propagadores de empleo, inversión extranjera directa (IED), transferencia de tecnología, efectos de proveeduría regional y nacional, vínculos y cadenas industriales y comerciales, y, en general, que puedan propiciar la transformación de la economía local para incidir en la región y en la economía nacional como un todo; para ello se les dotó de cierta autonomía de gobierno para introducir la legislación pertinente para atraer IED (Clauss, 2000:21).

\section{Inserción de China a la economía mundial y apertura gradual}

Cuando el gobierno central en China decide reformar el proyecto nacional y plantearse una política de apertura al exterior, prevalecían aún los viejos arreglos institucionales. Se sabía de la necesidad de cambios a fin de lograr mayores niveles de crecimiento económico y bienestar social; sin embargo, no había un proyecto claro ni un plan preconcebido que definiera un rumbo definido y único para toda China. La frase: "caminar a tientas sobre piedras mientras se cruza el río", de Deng Xiaoping, reflejaba en mucho esa incertidumbre. Se percibía la necesidad de transformar el sistema de planeación central tradicional en un sistema de mercado y de abrirse al exterior. Sin embargo, no se tenía la seguridad de las consecuencias que tendrían la aplicación de las políticas de apertura y del cambio institucional. De ahí el pragmatismo y la práctica experimental e incremental que caracterizó la aplicación de la política de puertas abiertas. En el mismo sentido, el papel del gobierno durante este periodo fue de supervisor, guía e inductor del proceso.

En consideración a esa dinámica y en orden para avanzar en la aplicación de la reforma en lo que se refiere a la apertura de la economía al resto del mundo, el gobierno chino decidió poner en marcha una política de puertas abiertas con el establecimiento 
de zonas económicas especiales. ${ }^{1}$ Durante esta primera fase, ni el gobierno central ni los gobiernos locales tenían una idea clara acerca del grado de apertura al mercado que debería adoptarse o de cómo reformar la economía centralmente planificada. "Las ZEE serían la primera experiencia que debería servir de catalizador para avances (o retrocesos) futuros" (Cornejo, 1985:20).

En agosto de 1980 el gobierno chino decide el establecimiento de cuatro zonas económicas especiales, Xiamen, Shantou, Shenzhen y Zuhai, con un doble propósito: ser laboratorios para experimentar sus políticas de la reforma económica y servir como ventanas al mundo exterior (Lau Raymond, 2001:26). Xiamen se estableció en la provincia de Fujián, mientras Shantou, Shenzhen y Zuhai se fijaban en la provincia de Guangdong. A diferencia de otras regiones, en ellas se impusieron medidas de mercado y a los gobiernos locales se les concedió autoridad para tomar decisiones sobre inversión pública, adicional a la planificación estatal. Se les permitió decidir políticas preferenciales para atraer IED, promover sus propios planes de infraestructura, así como decidir sobre la obtención de fondos para financiar proyectos de inversión (Kuotsai Tom Liou, 1999:194). El éxito en la aplicación de esas políticas no fue inmediato, pero Shenzhen, Xiamen, Zuhai y Shantou, en cada caso, incrementaron notablemente sus flujos de IED a partir de 1984, cuando se eliminan las distorsiones que les impedían en un principio lograr los objetivos para los que fueron creadas (Lau Raymond, 2001:32).

En 1984, el gobierno extendió su política de puertas abiertas a otras ciudades y provincias: se abrieron 14 ciudades costeras: en Shangai, GuangZhou, Tianjin, Dalian, QinhuangDao, Yantai, QingDao, LianYungang, Nantong, Ninbo, Wenzhou, Fuzhou, Zhanjiang y Beihai (Choosin, 1996); después se establecieron áreas de desarrollo económico y se incorporaron otras ciudades y provincias a estos esquemas de desarrollo. En 1988 el Consejo Estatal acordó transformar la isla de Hainan como la ZEE más grande de China y agrandar las otras cuatro; decidió también extender el establecimiento de ciudades costeras abiertas en las deltas de los ríos Perla (DRP) y Yangtze (DRY). En junio de 1990 el distrito de Pudong fue declarado ZEE, y se decidió que Shanghai sería la llamada "Cabeza del Dragón” (China in Brief, 2000). Desde 1992 el gobierno chino ha establecido zonas abiertas en las ciudades costeras con el Pacífico; se han agregado 15 zonas de libre comercio, 32 zonas de desarrollo económico y tecnológico y 53 zonas de desarrollo industrial para nueva y alta tecno-

1 Una zona económica especial se caracteriza por ser una unidad administrativa, disponer de un área de gran extensión territorial, ser un espacio geográfico abierto al mundo, disponer de una localización privilegiada y estar sujeta a la aplicación de políticas preferenciales. 


\section{Cuadro 1}

Número y formas deZEE en China

\begin{tabular}{lr}
\hline Zona de Desarrollo Científico Tecnológico & 56 \\
Zona de Desarrollo Industrial y de Tecnología Avanzado & 53 \\
Zona de Procesamiento para la Exportación & 15 \\
Zona de Cooperación Económica en la Frontera & 14 \\
Zona de libre Comercio & 12 \\
Zona de Desarrollo Turístico & 10 \\
Zona de Inversión en Taiwán & 4 \\
\hline Total & 164 \\
\hline
\end{tabular}

Fuente: Hernández Pedraza, 2005:95.

logías en ciudades grandes e intermedias (Gareth Powel, 2008). En 2006 la Nueva Área de Binhai, localizada entre Beijing y Tianjin, fue aprobada como la más reciente ZEE (Guo Wanda and Yueqiu Feng, 2007).

Aunque estas áreas adoptaron políticas preferenciales diferenciadas, han jugado un papel dual: por un lado, atraer IED que les permitiera, no sólo el acceso de capitales del exterior para elevar su planta productiva, sino que además y —quizá lo más importante- que los inversionistas trajeran la tecnología y el know how en el manejo de las empresas que les permitiera aprovechar sus capacidades para competir. Finalmente, habría que agregar que con el desarrollo rápido de las ZEE se pretendía el establecimiento de puentes con las regiones más atrasadas para posibilitar su crecimiento y desarrollo económico y generar efectos propagadores (Handfield \& McCormack, 2005).

\section{El desarrollo de Shenzhen como zona económica especial}

Shenzhen está situada en la provincia de Guangdong, costa sureste de China, adyacente a la bahía de Dapeng en el este y al norte de Hong Kong. Su extensión territorial aproximada es de 2,000 $\mathrm{km}^{2}$. Se divide en seis distritos urbanos: Baoan, Nanshan, Futian, Luohu, Yantian y Longgang. Cada uno de esos distritos se ha reconvertido en función de una división del trabajo regional y de producción de conocimiento al estilo smithiano (OCDE, 2000:22) sobre la base de sus ventajas de localización y en la disponibilidad de sus recursos naturales, capital humano y financiero. Nanshan es el centro donde se ubican industrias de alta tecnología; en Futian reside el gobierno municipal y se ubica en el corazón de la ZEE; Luohu, adyacente a Hong Kong, es el centro comercial y financiero, y Yantian se distingue por su logística. El puerto de Yantian tiene la segunda terminal de contenedores de agua profunda más grande de China y cuarta en el mundo.

El área del Delta del Río Perla fue la primera en aplicar la política de puertas abiertas iniciada en 1979. De las primeras cuatro ZEE, dos fueron de esta área: Shenzhen 
Cuadro 2

IED y su distribución, por región en China, 2006

\begin{tabular}{lcccc}
\hline Región & Número de proyectos & Participación & Valor realizado & Participación \\
\hline Total & 41,485 & 100.0 & 694,68 & 100 \\
Este & 35,538 & 85.6 & 569.22 & 81.94 \\
Central & 4,084 & 9.84 & 39.22 & 5.65 \\
Oeste & 1,851 & 4.46 & 21.7 & 3.13 \\
Otros & 12 & 0.03 & 64.47 & 9.28 \\
\hline
\end{tabular}

Región este: Beijing, Tianjin, Hebei, Liaoning, Shanghai, Jiangsu, Zhejiang, Shandong, Guandong Hainan.

Región central: Shanxi, Jilin, Heilongjiang, Jiangxi, Henan, Hubei, Hunan.

Región oeste: Inner Mongolia, Guangxi, Sicuani, Chongqing, Guizhou, Yunnan, shaanxi, Gansu, Qinghai, Xinjiang, Tibet.

Fuente: Invest in China (2007), "FDI utilized by East, Central and West Parts of China in 2006".

y Zhuhai. En 1985 la DRP fue declarada "región económica abierta" A principios de 1990 fueron aplicadas en la DRP las más intensas políticas de apertura al mercado en toda China a fin de integrarla con mayor rapidez a la economía global. Desde entonces esta región ha emergido como una de las más ricas en China (Lachang Lu and Yegua Dennos Wei, 2006:231). Debido a la proximidad con Hong Kong y a las políticas aplicadas, Shenzhen fue la ciudad de la DRP que se desarrolló con mayor rapidez con repercusión en Guangdong y en la región costera de China (véase el cuadro 2).

En 1980, Shenzhen era un pueblo pesquero con cerca de 30,000 habitantes, en 2008 su población registraba cerca de 10.5 millones de personas y en la actualidad es el principal centro productivo del país, el mayor receptor de IED y la ciudad comercial más activa que cualquier otra en China (Yaolin Mark y Xiaochen Meng, 2004). Su tasa promedio anual de crecimiento del PIB entre 1980 y 2006 fue de casi 22\%. Este crecimiento puede dividirse en tres etapas, como se observa en el siguiente cuadro. En cada una de éstas se observa una transformación estructural importante.

Cuadro 3

Etapas de crecimiento económico en Shenzhen

\begin{tabular}{|c|c|c|}
\hline Primera etapa & 1980-1986 & $\begin{array}{l}\text { Inicio de transferencia desde Hong Kong de industrias intensivas en trabajo. Se registra una tasa de } \\
\text { crecimiento promedio anual de } 44 \% \text {. En este periodo se realiza una fuerte inversión estatal en } \\
\text { infraestructura e instituciones educativas para generar los recursos humanos necesarios para las labores } \\
\text { administrativas y de gestión. }\end{array}$ \\
\hline $\begin{array}{l}\text { Segunda } \\
\text { etapa }\end{array}$ & 1987-1995 & $\begin{array}{l}\text { Se internacionaliza la inversión a Shenzhen. Pero de forma simultánea empieza a perder ventajas } \\
\text { comparativas en la producción de bienes intensivos en trabajo. El PIB crece a una tasa anual de } 29 \% \text { de } \\
\text { crecimiento promedio anual; el gobierno municipal impulsa un sistema de innovación científica y } \\
\text { tecnológica centrado en las empresas, con la participación de universidades e institutos de investigación y } \\
\text { desarrollo tecnológico e instituciones financieras. }\end{array}$ \\
\hline Tercera etapa & 1996-2006 & $\begin{array}{l}\text { Reconversión in dustrial de Shenzhen hacia la producción de bienes de alta tecnología y de servicios de alto } \\
\text { valor agregado. Su tasa de crecimiento promedio fue de } 19 \% \text { de } 1991 \text { a } 2000 \text {, la tasa de crecimiento } \\
\text { anual de industrias de hi-tech fue de } 53.3 \% \text {. En } 2000 \text { el valor dela generación de productos de hi-tech fue } \\
\text { de } 06.4 \text { billones de RMB yuanes, (precios corrientes), lo que hizo crecer en } 45.9 \% \text { el valor bruto de la } \\
\text { producción industrial. }\end{array}$ \\
\hline
\end{tabular}

Fuente: Elaboración propia con base en Guo Wanda y Yueqiu Feng, 2007. 
1. En la primera de etapa (1980-1986) el gobierno central estableció infinidad de oficinas como "ventanas" para "guiar", "supervisar" y "apoyar" a la IED y al comercio. Debido a la inversión gubernamental para desarrollar las funciones administrativas, el crecimiento registrado no se debió sólo a la IED, sino a la absorción de una gran cantidad de recursos del gobierno central. Durante estos primeros años los inversionistas extranjeros estaban a la expectativa de lo que sucedería con la nueva reforma ${ }^{2}$ (Lachang Lu y Yegua Dennos Wei, 2007).

2. Durante la segunda etapa (1987-1995) las transformaciones que se sucedieron en China obligaron a Shenzhen a afrontar nuevos retos. La política de puertas abiertas se hizo extensiva a otras áreas y regiones. Shenzhen, que en una primera etapa se había visto favorecida con esas políticas, empezó a perder gradualmente sus ventajas comparativas, ya que tuvo que compartir y competir con otras provincias y zonas costeras abiertas. Aunado a ello, los costos de los factores productivos (tierra, capital y trabajo) se incrementaron y el rápido crecimiento urbano y el deterioro ambiental de la industria productora de bienes intensivos en trabajo obligaron al gobierno local a buscar nuevas estrategias de crecimiento y desarrollo industrial. El gobierno municipal de Shenzhen se planteó transformar la ciudad en una ciudad de innovación (Lachang Lu y Yegua Dennos Wei, 2007: 235).

3. En la tercera etapa (1996-2006) se aprecia el éxito de las políticas de reconversión industrial hacia la producción de bienes de alta tecnología iniciado durante la etapa previa y cuya finalidad era sobre todo impulsar a la región, con base en una estrategia sustentada en la producción de conocimiento (Elliasson, 2000:148149). Como se observa en el cuadro 3.

Con este proceso de reforma y cambio estructural, Shenzhen pasó de ser una industria tradicional a principio de la década de los ochenta a una ciudad industrial de alta tecnología, gracias a la rápida reconversión tecnológica de sus empresas, que pasaron de ser productoras de bienes ensamblados a productoras de bienes manufacturados en su totalidad (Guo Wanda y Yueqiu Feng, 2007). Estas políticas tuvieron de inmediato sus efectos positivos: de 1991 a 2000 la tasa de crecimiento anual de industrias de hi-tech fue de 53.3\%. Y para 2005 el sector de las telecomunicaciones, computadoras y electrónica participó con más de $70 \%$ del total de la producción industrial, seguido por el sector eléctrico y maquinaria con $8.33 \%$, instrumentos y equipos de oficina $3.8 \%$, productos metálicos $3.16 \%$ y plásticos con $2.7 \%$ (Enright Michel J. 2007).

2 En esta primera fase cerca de $60 \%$ de la inversión provino del gobierno central. 
Cuadro 4

Shenzhen

Participación porcentual de las principales industrias según

la producción industrial en 2005 (\%)

\begin{tabular}{lr}
\hline 1. Equipo de telecomunicaciones, computadoras y electrónica & 70.02 \\
2. Equipo eléctrico y maquinaria & 8.33 \\
3. Instrumentos y e equipo de oficina & 3.87 \\
4. Productos metálicos & 3.16 \\
5. Productos de plástico & 2.77 \\
6. Otras manufacturás incluyendo artesanías & 2.21 \\
7. Equipo para propósitos especiales & 1.78 \\
8. Productos químicos y materiales químicos no refinados & 1.63 \\
9. Medios de impresión y grabación & 1.50 \\
10. Equipo de transporte & 1.33 \\
\hline
\end{tabular}

Fuente: Enright Michel, The Greater Pearl River Delta, Invest in Hong Kong, Hong Kong, 2007

Las compañías privadas generaron $21 \%$ de la producción total en 2006, entre ellas destacan transnacionales como Ricoh, Toshiba, Epson, Copier, Xerox, Wal Mart, Compaq, Sony, Intel, IBM, Siemens, Samsung, Mitsubishi, Mitsui, HP, AT\&T, Flextronic, Olympus, etcétera. De las 500 empresas multinacionales de mayor producción mundial, 141 tenían filiales en Shenzhen. El desarrollo de la industria tecnológica en Shenzhen se ha difundido hacia firmas locales, las que gradualmente han establecido sus propias marcas. Para fines de 2005 había cinco marcas propias de Shenzhen cuyas ventas habían superado los 1.25 billones de dólares. Muchas marcas conocidas han emergido en las áreas de telecomunicaciones, computación y productos relacionados, audio y video digital, software y biomedicina (Guo Wanda y Yueqiu Feng, 2007:7).

La IED captada por Shenzhen ha pasado de 153.7 millones de dólares en 1979, a 3.3 mil millones de dólares en 2006. Hong Kong ha sido la fuente más importante de capitales, al contribuir con las dos terceras partes del total de su inversión debido a su privilegiada localización y los vínculos culturales. Dicha IED genera una tercera parte de la fuerza de trabajo total, contribuye con tres cuartos del valor de la producción industrial total y genera cerca de $60 \%$ de las importaciones y exportaciones totales de la provincia de Guangdong (State Statistical Bureau, 2006). En 2006, el PIB de Shenzhen era de 71.3 miles de millones de dólares, cuarto entre las ciudades de mayor crecimiento en China y muy cerca de Guangzhou, tercera ciudad en importancia, y casi la mitad de Hong Kong. Por lo anterior, Shenzhen puede ser considerada como una de las ciudades que mayor impacto positivo ha tenido sobre el desarrollo regional (Taverner James, 2007; Cornejo, 2007; 92).

Por otra parte, para apuntalar el éxito en la transformación industrial, el gobierno municipal de Shenzhen continuó jugando un papel primordial. En el $11^{\circ}$ Plan Quinquenal 2006-2010 de la RPC, logró que se considerara prioritaria la transformación 
Cuadro 5

Indicadores económicos relevantes de la Delta del Río Perla (2006)

\begin{tabular}{lcccccccc}
\hline \multicolumn{1}{c}{ Civdades } & Área $\left(\mathrm{km}^{2}\right)$ & Población $(\mathrm{mn})$ & $\begin{array}{c}\text { PIB (RMB } \\
\text { bn) }\end{array}$ & PIB (\%) & $\begin{array}{c}\text { PIB per cápita } \\
\text { (RMB) }\end{array}$ & $\begin{array}{c}\text { Valor } \\
\text { industrial } \\
\text { (RMB bn) }\end{array}$ & $\begin{array}{c}\text { Export } \\
\text { (USSbn) }\end{array}$ & $\begin{array}{c}\text { Actual IED } \\
\text { (USSbn) }\end{array}$ \\
\hline Guangzhou & 7,434 & 9.95 & 607.4 & 14.8 & 63,100 & 197.1 & 32.4 & 2.9 \\
Shenzhen & 1,953 & 8.46 & 581.4 & 16.6 & 69,450 & 308.7 & 136.0 & 3.3 \\
Zhuhai & 1,688 & 1.24 & 74.8 & 16.4 & 52,185 & 41.5 & 14.8 & 0.8 \\
Foshan & 3,849 & 5.34 & 292.8 & 19.3 & 50,232 & 174.4 & 21.1 & 1.1 \\
Huizhou & 11,158 & 3.22 & 93.5 & 16.6 & 25,043 & 40.4 & 12.3 & 1.0 \\
Dongguan & 2,465 & 6.45 & 262.7 & 19.2 & 39,468 & 131.9 & 47.4 & 1.8 \\
Zhongshan & 1,00 & 2.36 & 103.6 & 16.8 & 42,058 & 69.6 & 15.6 & 0.7 \\
Jiangmen & 9,451 & 3.95 & 94.2 & 15.6 & 22,936 & 43.9 & 7.4 & 0.7 \\
Zhaoging & 14,856 & 3.38 & 51.6 & 14.5 & 13,991 & 11.4 & 1.8 & 0.7 \\
\hline
\end{tabular}

Fuente: Guangdong Statistical Yearbook, 2007.

definitiva de la industria hacia la alta tecnología (Hi Tech) y servicios de alto valor (Enright Michel J., 2007). En congruencia con tal propósito, la inversión municipal en ciencia y tecnología alcanzó 1.5 billones de RMB (también reconocidos como yuanes) en 2006, 7.26\% de la inversión pública total de la ciudad. La inversión en ciencia y tecnología alcanzó 92.5 billones de RMB, aplicándose 3.65\% del PIB para investigación y desarrollo tecnológico (Lachang Lu and Yegua Dennos Wei, 2007:235).

\section{Factores que explican el éxito de Shenzhen}

Sin lugar a dudas, muchos son los factores que explican su éxito, sin embargo los que consideramos más relevantes son:

1. El primero y el más importante radica en que el gobierno central aplicó su política de puertas abiertas y su reconversión como ZEE. Fue en Shenzhen donde se llevó a cabo por primera vez, y con mayor amplitud, una reforma comprensiva de mercado. Como ZEE, disfrutó de un esquema de políticas preferenciales. Ello le permitió crear condiciones favorables para atraer capitales del exterior y estimular la competitividad de las empresas. ${ }^{3}$ La política de puertas abiertas respondió a un momento determinado y a circunstancias particulares. Aunque fue el inicio de grandes cambios, se tuvo que ir reconvirtiendo las políticas preferenciales de la ZEE en función de la situación cambiante del entorno y de los objetivos prioritarios de crecimiento económico establecidos por los gobiernos central y local.

2. Shenzhen disfruta de una ventaja privilegiada en su localización al ser una ciudad costera adyacente a Hong Kong. Shenzhen está localizada frente a la DRP y

3 La IED se incrementó $10 \%$ anual de 1980 a 2006. El valor de las exportaciones alcanzó la cifra de 136.1 billones de dólares en 2006. 
conectada a Hong Kong en el sur. Su ubicación geográfica es estratégica, por su cercanía con Hong Kong y por servir de puente para las transacciones económicas y comerciales de China continental con el mundo capitalista. La estructura industrial ascendente y la competitividad de las empresas de Shenzhen se han visto favorecidas por esta proximidad con Hong Kong, de donde provienen, no sólo los montos más importantes de capital para inversión, sino todo el know how empresarial, comercial y tecnológico. En una primera fase, a principios de la década de los ochenta, dada el alza en los costos de producción local y la proximidad geográfica y cercanía cultural, los empresarios de Hong Kong decidieron transferir sus industrias intensivas en trabajo a la DRP, en particular a Shenzhen; después fueron transferidas empresas con mayor tecnología y de servicios (Lachang Lu y Yehua Dennis Wei, 2006:233).

3. El papel del gobierno local como promotor del crecimiento y desarrollo económico. Dadas las circunstancias particulares de China, durante la primera fase de la aplicación de las políticas de puertas abiertas (1980-1990) el gobierno central jugó un papel fundamental en la aplicación de la reforma económica y, por ende, fue el responsable de los avances obtenidos. Desde ahí se decidía y aplicaba todo lo referente a la política de puertas abiertas. Mientras tanto, los gobiernos locales se sujetaban a lo dispuesto desde Beijing, de manera tal que sus documentos y decisiones eran como "copias al carbón" de lo dispuesto desde el centro (Yaolin Mark y Xiaochen Meng, 2004:185). Debido a ello, las políticas aplicadas en Shenzhen, al igual que el resto de las ZEE, habían estado sujetas a lo dispuesto desde el poder central. A partir de 1992, posterior al tour emprendido por Deng Xiaoping hacia las regiones de la costa, se autorizó al gobierno municipal de Shenzhen para que pudiera legislar. El establecimiento de nuevas regulaciones y leyes emitidas por el gobierno municipal no fueron simplemente una nueva legislación, sino una nueva práctica que buscó crear un ambiente propicio para la inversión local de multinacionales y generar mayores incentivos, sobre todo para las inversiones de alta tecnología. Lo más importante de todo estriba en que el sistema legal y las nuevas regulaciones se decidieron en función de los requerimientos y necesidades locales, no tanto de los intereses nacionales o transnacionales (Yaolin Wang y Xiaochen Meng, 2004: 186-87). Por tanto, fue la capacidad en la toma de decisiones en el gobierno municipal lo que les permitió establecer un sistema legal propicio y aplicar políticas preferenciales propias a fin de alentar la inversión de empresas de hi tech y desestimular inversiones no acordes con los objetivos prioritarios para la ciudad. 
4. Importancia de la infraestructura urbana. Shenzhen fue objeto de importantes inversiones gubernamentales en infraestructura portuaria y urbana, lo que le ha permitido alcances extraordinarios. Es la única ciudad china que dispone de puertos de varios tipos, aeropuertos y marina. El puerto de Yantian en Shenzhen se ubica como la segunda terminal de contenedores de agua profunda más grande de China y cuarto en el mundo. Por otro lado, Shenzhen es de las ciudades con más y mejores carreteras y vías férreas que la conectan con las principales ciudades de la DRP y con Hong Kong. Los gobiernos central y municipal son conscientes de que una buena infraestructura es necesaria para crear un ambiente logístico favorable que permita incrementar la competitividad industrial de la ciudad.

5. Shenzhen ha sido casa de emigrantes provenientes del interior del país y más recientemente del exterior. El rápido crecimiento económico alcanzado y las políticas inmigratorias asumidas por el gobierno central para ofertar una fuerza de trabajo de bajo costo han transformado a Shenzhen en un polo de atracción de grandes montos de población joven del interior. Esta ciudad no sólo es un mosaico cultural que combina la cultura diversa de otras provincias y regiones de China, sino que además ha incorporado rasgos del exterior de China, debido a la fuerte influencia de Hong Kong y al arribo de un gran número de empresarios e inversionistas de diversas partes del mundo. Esta circunstancia ha permitido que la población de origen chino en Shenzhen disponga de una cultura que, sin abandonar los rasgos propios de su cultura, asimila lo mejor de otras, como es la actitud empresarial y la disposición al ahorro y a la innovación (Fang, T., 2001).

\section{Políticas claves que han incidido para la competitividad de Shenzhen}

a) Una de las políticas claves que explican la transformación industrial de Shenzhen ha sido la habilidad del gobierno para atraer inversión del exterior (IE), lo que le ha permitido transformar la estructura industrial y hacerla más competitiva. Las empresas extranjeras participan con $40 \%$ del PIB total de Shenzhen. Entre las principales medidas para atraer IE se pueden considerar la eliminación de trabas burocráticas y administrativas a la inversión extranjera; facilitación de los permisos para el establecimiento de compañías de propiedad total de extranjeros; autorización para el libre acceso a la tierra; dotación de la infraestructura requerida por las firmas extranjeras para el desarrollo de su actividad productiva en parques industriales; permiso para la repatriación de las utilidades; el establecimiento de políticas favorables para la importación de bienes tecnológicos; apoyo logístico 
y financiero para empresas con capacidad exportadora; reducción o excepción de tasas impositivas en función del tipo de compañía; deducción impositiva debido a gastos de entrenamiento y capacitación de personal; apoyo a los trabajadores con escuelas y guarderías para sus hijos, y establecimiento de fondos gubernamentales para investigación y desarrollo tecnológico (Shenzhen Government, 2008).

b) La transformación de Shenzhen en función de la creación de nuevas ventajas competitivas. Por tradición, la economía de Shenzhen había dependido de la IED de la industria intensiva en trabajo, cuyo crecimiento fue sin precedentes durante la década de los ochenta e inicios de la de los noventa. Ya a mediados de esta última su modelo de crecimiento dejó ver serias limitaciones, sobre todo el de su baja productividad, por lo que el gobierno decidió llevar a cabo acciones con la finalidad de transferir ese modelo de crecimiento a otro centrado en la ciencia y la tecnología. Valorando sus fuerzas y debilidades externas e internas, el gobierno elaboró la Estrategia para el desarrollo científico y tecnológico y el Perfil para el desarrollo de investigación de nueva y alta tecnología. Como parte importante de esta estrategia Shenzhen fue denominada "Ciudad Nacional de Innovación" y se decidió y apoyó el establecimiento de algunas zonas de hi tech (Lachang Lu y Yegua Dennos Wei, 2006:237). Para lograr la transformación industrial, se aprobó que 4\% del PIB de la ciudad fuese utilizado para alentar la investigación y el desarrollo tecnológico ${ }^{4}$ (Lachang Lu y Yegua Dennos Wei, 2006:235).

c) En apoyo a la política de creación de nuevas ventajas comparativas, se carecía de personal altamente capacitado y la formación local era insuficiente para lograr la transformación de la estructura productiva hacia el sector de hi tech, por lo que en 2001 se persuadió a los graduados de las principales universidades e institutos en China a establecer un centro universitario ${ }^{5}$ con la calidad necesaria para la formación de técnicos calificados y la generación de investigación y desarrollo tecnológico. Dicho centro universitario se creó en 2003 con científicos e investigadores provenientes de las tres principales universidades de la región (Universidad de Pekín, Universidad de Tsinghua, ambas en Beijing, y del Instituto de Tecnología en Harbin); para ello fue necesario darles facilidades de acceso, fondos generosos y un ambiente propicio para el desarrollo de sus actividades.

d) La concentración urbana obligó al gobierno municipal a desconcentrar la actividad productiva a espacios geográficos con menor población y aprovechar los

4 Con esa inversión se espera que la producción de hi tech se incremente a un promedio anual de $20 \%$ en los próximos años.

5 Antes de esta fecha había en Shenzhen una universidad con sólo 25 estudiantes graduados, los que eran, por supuesto, insuficientes para hacer de ella una ciudad moderna. 
centros urbanos para la realización de operaciones industriales con mayor valor. En este sentido, los gobiernos locales y provinciales aplicaron políticas para el establecimiento de empresas multinacionales de industrias intensivas en tecnología y se actuó para transformar a Shenzhen en una ciudad donde se concentrara el poder de mando de las grandes empresas que operan en China (Guo Wanda y Yueqiu Fena, 2007). Los resultados de estas políticas están a la vista: desde principios de los noventa las empresas transnacionales expandieron sus áreas de inversión de manera que el establecimiento de centros de investigación y desarrollo tecnológico hicieron de esa región uno de los tres centros de investigación y desarrollo más importante de toda China (los otros dos son Beijing y Shangai). ${ }^{6}$

e) Desarrollo de un sistema financiero moderno y eficiente. Para dar seguridad y eficiencia a la actividad productiva de las empresas privadas domésticas y multinacionales, el gobierno de Shenzhen estableció un sistema financiero integral con un moderno sistema bancario, fianzas y seguros. Todas las instituciones financieras domésticas tienen sucursales en Shenzhen y todos los bancos de esta localidad tienen cobertura mundial para sus operaciones, además de que cerca de 50 instituciones financieras extranjeras han establecido sucursales en esta ciudad. Una de las dos operadoras de bolsa establecidas en China está localizada en Shenzhen.

f) En el sentido de concentrar a las grandes empresas y conglomerados industriales el gobierno local se dio a la tarea de alentar la formación de clusters industriales o concentraciones industriales para beneficiarse de las economías de escala y mejorar las ventajas comparativas regionales. Debido a este tipo de organización industrial se ha generado un enlace productivo y financiero eficaz entre las empresas, posibilitando con ello asegurar el abastecimiento y responder con eficacia a las exigencias del mercado. Los principales clusters en Shenzhen son de electrónica, computadoras, telecomunicaciones, juguetes, plástico, relojes, pinturas de óleo, servicio postal, servicios de logística, servicios portuarios, finanzas, árboles artificiales e impresoras (Market Profiles, 2007).

6 Para ilustrar los avances obtenidos basta con mencionar la experiencia de Nokia. Esta firma multinacional inició operaciones en el distrito de Gongguang en 1995, nueve años después (2004) se producía en este distrito de Shenzhen $80 \%$ de todos sus productos que se exportaban a los países de occidente. Para 2004, 10 empresas transnacionales habían establecido en Shenzhen sus centros de investigación y desarrollo tecnológico (Lachang Lu y Yegua Dennos Wei, 2006:235) 


\section{Lecciones para México de las ZEE en China:}

\section{el papel del Estado en las transformaciones institucionales}

A diferencia de China, cuya reforma fue dirigida por un Estado fuertemente vertebrado y autoritario, en México el gobierno consideró que el fracaso de las estrategias económicas del modelo de sustitución de importaciones se debió a una intervención excesiva del Estado en la economía, la cual fue determinante para las apariciones de las crisis económicas de mediados de la década de los setenta y sobre todo la de 1982. Debido a lo antes expuesto, desde los inicios de la década de los ochenta en México se aplicó un modelo y una estrategia de economía abierta. Ésta se llevó a cabo sobre la base de cuatro ejes fundamentales: sustitución de la rectoría económica del Estado por el automatismo del mercado en todos los ámbitos posibles; reducción y, de ser posible, eliminación de la intervención económica del Estado en la economía mediante la desincorporación parcial o total de las empresas públicas; puesta en marcha de la apertura económica y desregulación de la economía en todos los ámbitos posibles (Ugarte, Fernando, 1994:79-122).

De esta manera, el gobierno mexicano de las últimas tres décadas ha impulsado políticas económicas que reafirman la menor intervención estatal en la economía. Congruentes con esta estrategia, se aplicaron medidas de apertura y desregulación económica con un claro objetivo: fomentar la participación del sector privado y atraer ahorro del exterior. Con ello, les cedieron a los actores privados la preeminencia económica, con lo que la acción del Estado quedó restringida a la esfera macroeconómica y se evitó, en lo posible, el impacto de agentes externos en el quehacer económico (Ibarra, 2005; 107-120).

Aunque México y la República Popular China aplicaron sus respectivas estrategias casi en un mismo momento histórico y de que se plantearon propósitos similares, inserción a la economía mundial, los resultados fueron contrastantes. China con su política intervencionista dio un rumbo más definido a su economía, sus indicadores económicos son sólidos y presenta una gran credibilidad económica y política. México, en cambio, sujeto a las fuerzas del mercado y con una cada vez menor intervención del Estado en la economía, mantiene indicadores zigzagueantes, no se ha logrado un crecimiento económico consistente y su credibilidad económica está en duda (González, 2002:85).

Las decisiones de política económica se han hecho en función del modelo económico nuevo liberal, en el que el gobierno no se compromete con acciones directas en el quehacer económico en su conjunto y deja las principales decisiones de política económica en los sectores privados. De hecho, no se reconoce la gradualidad en la 
aplicación de políticas, ni mucho menos se formula una política industrial definida con acciones específicas con base en un proyecto de desarrollo de largo alcance (González y Meza Lora; 2008;775), excepto del establecimiento de medidas de apoyo a los sectores privados, en quienes recae la verdadera toma de decisiones; el gobierno mexicano busca no meter las manos en la priorización de sectores. De manera implícita se reconoce la regulación automática del mercado, cuando la rígida realidad muestra, una y otra vez, que las economías de mercado no se regulan por sí mismas y de que no se puede dejar a la economía en "piloto automático" (valga la expresión), mucho menos, cuando éstas funcionan con "fallas" evidentes. Dejar que las fuerzas del mercado actúen por sí solas y sean las que conduzcan la economía significa no tomar lección alguna de otras economías.

\section{El establecimiento de una estrategia de desarrollo desigual}

La estrategia de desarrollo seguida por México radicó en introducir un nuevo modelo de desarrollo económico "sin transición hacia el modelo exportador" (Villarreal, 2000: 678), esperando que la sola apertura externa y el imperio del mercado fueran suficientes. Se supuso que se reducirían las desigualdades regionales de manera simultánea con el fin de que todas las regiones aprovecharan los beneficios del proceso de inserción de México a los mercados internacionales. ${ }^{7}$ Sin embargo, lejos de reducirse las desigualdades regionales, éstas se han ampliado, lo que aporta un elemento más al fracaso de la estrategia de desarrollo regional equilibrado que busca México (Sánchez González, 2006:120) y pone en evidencia el hecho de que en la actualidad no es posible pretender el desarrollo regional desde una concepción pragmática de que el mercado resolverá las desigualdades regionales, pero excluyendo a los agentes económicos de la formulación de un plan para el desarrollo regional. En este sentido, para que cualquier estrategia de desarrollo regional pueda tener éxito, se requeriría plantear la hipótesis del interés y la participación común del Estado-mercado (Ruiz Durán; 1999; 300), ya que ambos deben jugar papeles mutuamente incluyentes.

El desconocimiento de otras estrategias congruentes e integrales con el modelo de desarrollo económico nacional, al intentar reducir de manera simultánea el desarrollo regional desigual, ha impedido la posibilidad de una estrategia que pudiese favorecer a las regiones con mayor potencial, e impide que haya políticas de promoción espe-

7 El objetivo 13 del Plan Nacional de Desarrollo 2007-2012, en su apartado sobre Desarrollo Regional, establece: "Superar los desequilibrios regionales aprovechando las ventajas competitivas de cada región, en coordinación y colaboración con actores políticos, económicos y sociales al interior de cada región, entre regiones y en el ámbito nacional”. 
cífica que otorguen medidas preferenciales que mejoren el potencial regional, que se dirijan recursos adicionales para infraestructura y otorgamiento de servicios para detonar su crecimiento, etcétera. Para ilustrar lo anterior, el ejemplo más cercano es el del establecimiento de ZEE en China y el de las zonas libres en México en el noroeste y sureste del país.

Cabe aclarar que antes que China, México aplicó zonas económicas de excepción y que el primero aprendió del segundo (como quizá lo hizo de otros tantos países, a los que envió visores en la década de los setenta para preparar su apertura al mundo) para la puesta en marcha de las suyas. Las estrategias para el establecimiento de zonas francas difirieron en China y México. Los líderes chinos aplicaron una estrategia de desarrollo desigual favoreciendo a las regiones de la costa conforme esa estrategia fue dando resultados positivos se fue extendiendo a otras ciudades y regiones, al grado de que hoy la mayor parte de la zona costera en China está completamente abierta a la IED y al comercio internacional. México, que había puesto en práctica zonas libres desde 1965 en las regiones fronterizas con Estados Unidos en el norte y Belice en el sur, a principios de la década de los noventa inició un proceso de gradual eliminación del llamado régimen de excepción a fin de incorporar a todo el país a un nuevo régimen impuesto por el TLCAN con Estados Unidos y Canadá. Aunque las zonas libres habían jugado un papel importante en el crecimiento y desarrollo económico de la región noroeste durante varias décadas, fueron desmanteladas con el fin de homogeneizar a las regiones en torno a una política nacional. Los resultados en la aplicación de ambas estrategias son evidentes: China se ha insertado con éxito a la economía mundial. Hoy México es más dependiente de la economía estadounidense, mientras las ZEE de China han alcanzado un crecimiento extraordinario y han logrado reconvertirse a fin de seguir aprovechando su despegue inicial y su privilegiada localización y ser el motor que arrastre a otras regiones y provincias del interior; en México, en aras de superar los desequilibrios regionales de manera simultánea, al desaparecer el régimen de zona libre, la región del noroeste no sólo no ha logrado reconvertirse, sino que al carecer de un plan preconcebido del quehacer futuro y al depender únicamente de las fuerzas del mercado, tiende a perder sus ventajas comparativas iniciales. Además, ha olvidado que con modelos económicos de apertura externa los sectores económicos clave y las regiones deben jugar un nuevo papel para hacer compatible y congruente la aspiración de aprovechar los fines de la estrategia de apertura externa con integración regional (Katz, 1999; 338).

Una China abierta a las economías del mundo y con un crecimiento importante de su demanda interna presenta mayores incentivos de inversión en sus ZEE que todo el 
territorio mexicano. El atractivo de la inversión en México, fundamentalmente en su región noroeste, muestra una preocupante tendencia, dada la pérdida de las ventajas comparativas y la agudización de la recesión reciente en el mercado estadounidense entre 2007 y 2009, con la creciente contracción de su demanda de bienes importados del exterior.

Para que en México cambiara la situación actual de fracaso del modelo y estrategia de desarrollo económico y de desarrollo regional, se requeriría de una política de desarrollo regional ad hoc a los fines del modelo de desarrollo económico, que articulara a ambos desde una amplia perspectiva: de integración regional, de cadenas productivas, política industrial, proyectos regionales, inversión en sistemas regionales de innovación, y de reconsideración de los objetivos de la apertura externa (Graizbord y Aguilar; 2006;112). Sólo de esa manera es posible esperar a que — de continuar el actual modelo de desarrollo económico secundario exportador o nuevo liberal— se dé la transición final entre un modelo de desarrollo basado en los mercados internos, con "desarrollo regional equilibrado" a otro con apertura externa y mercados externos, que - con una estrategia de "desarrollo regional desequilibrado" pero ad hoc a una estrategia de largo plazo y gradual- logre finalmente una inserción exitosa en la globalización económica mundial y cumpla con las metas del desarrollo económico y social.

\section{Consideraciones finales}

Del análisis realizado se deduce que la estrategia de REN y la inserción internacional o apertura externa son condiciones sine qua non para propiciar que un país tenga éxito en sus propósitos de desarrollo o disminución del subdesarrollo. En el caso de China, su REN y AE del territorio fueron dirigidas por el Estado, que ha sabido compaginar el gradualismo con el ensayo de prueba y error, para no cometer desaciertos de estrategia que puedan generar problemas internos, derivado de la inserción internacional del territorio a la economía internacional de hoy día, más interdependiente y competitiva. Lejos de buscar un desarrollo regional equilibrado, la estrategia del gobierno chino fue la de considerar un desarrollo desigual regional como punto de partida de su apertura al exterior. Shenzhen es una de las ZEE más exitosas de esta política. La aplicación de políticas desiguales le permitió a esa ciudad aprovechar sus recursos, potencialidades y ventajas, haciendo de ella uno de los centros más dinámicos y prósperos de China, lo que se fortaleció con la política de libertad y autonomía para legislar en favor del desarrollo económico y regional de la ZEE. 
La descentralización, la autonomía, la pertenencia a una región geográfica de importancia y el encadenamiento de Shenzhen a la región del territorio chino al exterior han sido los factores que han permitido a esta ZEE contribuir al acelerado crecimiento económico de la región oriental de China continental y ha sido detonante para generar eslabonamientos industriales y tecnológicos hacia el interior del país, fortaleciendo el crecimiento y desarrollo regional endógeno (Taverner, 2007:4). Es la ZEE de Shenzhen, fruto de un modelo de desarrollo económico, la que garantiza la articulación de los intereses nacional-regionales, con el interés de los agentes económicos del exterior, representados sobre todo por las corporaciones transnacionales. Así, el modelo económico chino expresa una combinación única de pragmatismo y visión que obedece a circunstancias históricas y culturales propias.

El caso de Shenzhen, como ZEE, es ilustrativo para México, país que - con un modelo de desarrollo económico orientado hacia el exterior, pero sin gradualismo y sin un horizonte de largo plazo- pensó que con la firma del TLCAN y la renuncia tácita a las zonas libres, francas o de excepción le generaría mayores beneficios. Shenzhen demuestra que sí es posible impulsar una determinada forma de inserción internacional, a partir de un modelo de desarrollo económico propio y que atienda a las necesidades regionales y nacionales de desarrollo económico.

En 2009, se cumple la vigencia del TLCAN, por lo que México aún puede intentar recuperar parte de su autonomía económica y reformular su estrategia de inserción internacional a partir de una estrategia global, que rediseñe su inclusión y reevalúe los beneficios de contar con ZEE. Su pertenencia a la Organización Mundial de Comercio no es obstáculo, ya que China es miembro y las aplica sin objeción alguna. Por otro lado, el cambio de gobierno en Estados Unidos en enero de 2009 ha generado expectativas para la revisión de dicho acuerdo, lo que de proceder, le brinda la oportunidad de rediseñar su estrategia global de desarrollo económico, por lo que no se debe dejar pasar esa oportunidad, pues la cambiante e incierta situación de la economía internacional obligan a una mayor flexibilidad y rapidez en la toma de decisiones y en la aplicación de sus políticas. Además, no se debe olvidar que el resurgimiento de las ZEE en el mundo es un indicador que bien puede ser tomado en cuenta para redefinir la forma de insertarse a la economía global en este siglo XXI. 
Bibliografía

Aguilar, Adrián Guillermo y Graizbord, Boris, "Regional Differences and the Economic, Social Geofraphy of Mexico at the Beginning of the Twenty-first Century" en Randall, Laura, Changing Structure of Mexico/ Political, Social, and Economic Prospects, Nueva York, Sharpe, 2006, pp. 91-118.

Bhaskar Goswami, "Special Economic Zones; Lessons from China", 13 de febrero 2007, en http://www.countercurrents.org/ goswami130207.htm.

Calva, José Luis, Educación, ciencia, tecnología y competitividad, México, Cámara de Diputados-Porrúa-UNAM, 2007.

China Economic Information Network, Statistics, 2007, http://ce.cei.gov.cn.

Choosin, T., "Foreign direct investment from the People's Republic of China", en Bettignies Henri-Claude de, Business transformation in China, Londres-Boston, International Thomson Business Press, 1996.

Cornejo B., Romer A., "Las Zonas Económicas Especiales en China”, en Estudios de Asia y Africa, vol. XX, núm. 2, México, Colegio de México, 1985, pp. 15-35.

"Nuevas Propuestas de Cambio en el Patrón de Desarrollo de China", en Calva, José Luis, Desarrollo económico: estrategias exitosas, México, Cámara de Diputados-Porrúa-UNAM, 2007, pp. 91-106.

, China. El Ascenso de una Potencia, México, Colegio de México, 2008.

Deng Xiaoping, "Nosotros podemos desarrollar una economía de mercado bajo el socialismo", Trabajos selectos de Deng Xioping, Beijing, People's Daily Online, 26 de noviembre, 1979, en http://english.peopledaily.com.cn/dengxp/.

Departamento de Comercio de Estados Unidos, "Shenzhen. Overview", en http://www.buyusa.gov/china/en/shenzhen.html.

Eingereicht von Claus Knoth, Special Economic Zones and Economic Transformation: The Case of the People's Republic of China, Germany, Konztanz, 2000.

Enright Michel J., "The Greater Pearl River Delta", Invest in Hong Kong, Hong Kong, 2007.

Fang, Tony, "Culture as a driving force for interfirm adaptation: A Chinese case", Industrial Marketing Management, vol. 30, núm. 1, Yale, Yale Center for the Study of Globalization, 1 de enero 2001.

Favorable Policies, Industry policies, Shenzhen Government Online, Shenzhen, 2008. http://english.sz.gov.cn/.

González García, Juan, "Reforma económica institucional y modelo de desarrollo con orientación externa: similitudes y diferencias entre México y la República Popular China (1980-2000), en Problemas del Desarrollo/ Revista Latinoamericana de Economía, vol. 33, núm. 129, México, IIEcUNAM, 2002.

, China: reforma económica y apertura externa. Transformación, efectos y desafios. Un enfoque neoinstitucional, México, Porrúa,. 2003.

, "Teoría del Desarrollo Económico. Situación Reciente y perspectivas", en Correa López, Gabriela y González García, Juan, Perfiles recientes del desarrollo económico de China, México, UAM-UdeC, 2006, pp. 17-49.

, "Lecciones del Proceso de Transformación Económica Institucional Reciente de la República Popular China", en Calva, José Luis (coord.), Desarrollo económico: estrategias exitosas, México, UNAM-Porrúa, 2007, pp. 107-121.

y Salvador Meza Lora, "Política Industrial y Nueva Economía Institucional en el Siglo XXI", en Comercio Exterior, vol. 58, núm. 11. México, 2008, pp. 669-679.

Guo Wanda y Yueqiu Feng, "Special Economic Zones and Competitiveness. A case Study of Shenzhen, the People's Republic of China”, PRM Policy, Serie 2, Islamabad, Noviembre de 2007.

Handfield, R. B., McCormack, K., "What you need to know about sourcing from China", The Journal of Supply Chain Management Review, vol. 9, núm.6, Newton, MA, Cahners Pub. Co, 2005.

HKTDC, "Yangtze River Delta", Market Profiles on Chinese Cities and Provinces, en http:// info.hktdc.com/mktprof/china/prd.htm.

Hernández Pedraza, Gladys et al., "Las Zonas Económicas Especiales. Algunas Experiencias", en Temas de la Economía mundial, La Habana, CIEM, vol. 8, 2005, pp. 88108. 
Ibarra, David, Ensayos sobre economía mexicana, México, FCE, 2005.

Katz, Isaac, "Efecto regional de la apertura comercial", Transición económica y comercio exterior, México, FCE-BANCOMEXT, 1997, pp. 325-358.

Kuotsai Tom Liou, "Strategies and lessons of China's Post- Mao Economic Development", Policy Studies Review, Florida, University of Central Florida Press, primavera de 1999.

Lachang Lu and Yehua Dennis Wei, "Domesticating Globalization, New Economic Spaces and Regional Polarization in Guangdong Province, China", Royal Dutch Geographical Society KNAG, vol. 98, núm. 2, Faculty of Sciences, Utrecht University, 2007.

Lau, Raymond W. K., "Pioneering Economic reform in China's Special Economic Zones", Brookfield, Singapore \& Sydney, Ashgate, 1999.

Lin, Justin Yifu, Fang Cai y Zhou LI, The China Miracle: Development Strategy and Economic Reform, Hong Kong, Chinese University Press, 1996.

Lu, L.C, "Polarization, new polarization and the development of the Pearl River Delta", citado por: Lachang Lu and Yehua Dennis Wei, "Domesticating Globalization, New Economic Spaces and Regional Polarization in Guangdong Province, China", Royal Dutch Geographical Society KNAG, vol. 98, núm.2, Faculty of Sciences, Utrecht University, 2007.

Meng Guangwen, "Experiences and Prospects of China's Free Economic Zones after over 20 Years", Chinese Business Review, vol. 4, núm. 9, Serial 27, septiembre de 2005.

$\mathrm{OECD}$, Knowledge Management in the Learning Society, París, 2000.

Opening to the Outside World, China in Brief, 2000, en http://mdjnkj.china.com.cn/e-china/openingup/sez.htm.

Ota, Tatsuyuki, "The Role of Special Economic Zones in China's Economic Development As Compared with Asian Export Processing Zones: 1979-1995", Asia in Extenso, March 2003 ( an on line research publication), 2005: www.iae.univ-poitiers.fr).

Park, Jung-Dong, The Special Economic Zones of China and their Impact on Its Economic development, Korea, Korea Development Institute, 1997.

Powell Gareth, China economic Review, Hong Kong, Sheung Wan, 22 de abril de 2008.

Ruiz Durán, Clemente, Macroeconomía Global/Fundamentos Institucionales y de Organización Industrial, México, Jus, 2000.

Sánchez González, Enrique, Economía mexicana para desencantados, México, FCE, 2006.

Shirk L. Susan, The Political Logic of Economic Reform in China, Berkeley, Series on Social Choice and Political Economy, University of California Press, 1993.

State Statistical Bureau, China Statistical Yearbook, Beijing, 2007.

Stoltenberg Clyde, "China's Special Economic Zones", Asian Survey, vol. 24, Berkeley, University of California Press, 6 de junio de 1984.

Tarverner, James, "El desarrollo económico en China y la influencia de las zonas económicas especiales", IX Reunión de Economía Mundial, Madrid, abril de 2007.

Ugarte, Fernando et al, La política industrial ante la apertura, México, FCE, 1994.

Villarreal, René, Industrialización, deuda y desequilibrio externo en México/Un enfoque macroindustrial y financiero 19292000, México, FCE, 2000.

Weiping Wu, Pioneering economic reforms in China's special economic zones: the promotion of foreign investment and technology transfer in Shenzhen, Brookfield, Ashgate Press, 1999.

Yaolin Wang Mark y Xiaochen Meng, "Global local initiatives in FDI: The experience of Shenzhen, China", Asia Pacific Viewpoint, vol. 45, núm. 2, Wellington, August 2004.

Yongtu Long, "China and the World economy", en Fourth Annual Stavros S. Niarchos, Lecture for Institute International Economics, Washington, DC, Peterson Institute, 23 de mayo de 2005.

Zhuang Guijun y Zhou, Nan, "The relationship between power and dependence in marketing channels: A Chinese perspective", European Journal of Marketing, Bradford, West Yorkshire, MCB Publications, vol. 38, 2004. 